\title{
Simplified Three-Cornered-Hat Technique for Frequency Stability Measurements
}

\author{
Lachlan J. Gunn, Student Member, IEEE, Peter G. Catlow, Waddah A. Al-Ashwal, Member, IEEE, \\ John G. Hartnett, Andrew Allison, and Derek Abbott, Fellow, IEEE
}

\begin{abstract}
We propose a novel technique allowing the use of the three-cornered-hat method with two devices under test (DUTs) and a time-tagging system that employs a common reference oscillator. The precision of a time-tagging system is reduced by fluctuations in the timebase, which are canceled when the relative phase between the two DUTs is measured. However, the raw timetags in this system provide a phase comparison between the DUTs and the system timebase, allowing the use of the three-cornered hat with some dual-channel measurement instruments.
\end{abstract}

Index Terms-Frequency stability, noise measurement, phase noise, test equipment, three-cornered-hat method.

\section{INTRODUCTION}

$\mathbf{H}$ IGH-STABILITY oscillators have become increasingly vital, perhaps most visibly in the global positioning system (GPS) [1], but also for such applications as telecommunications [2], astronomy [3]-[6], and radar [7], [8]. The characterization of these oscillators is vital, but doing so requires comparison with oscillators of similar stability [9] - a requirement that becomes far less onerous if the number of comparisons can be reduced.

The measurement of frequency stability is universally differential; an observer cannot simply listen to a clock and say that it has ticked 3601 times this hour, as it is then necessary to first determine precisely when an hour has passed. Such measurements contain instability from both sources, providing a variance that is the sum of those of the individual devices.

The three-cornered-hat method [10] can separate the individual variances from the combined measurements, taking advantage of the fact that, when the number of independent sources $M$ increases, the number of possible comparisons $1 / 2 M(M-1)$ increases quadratically. Then, if three sources are available, the $3 \times 3$ system of linear equations relating the source and measured variances can be solved for the performance of the individual devices. In this case, the source

Manuscript received July 26, 2013; revised September 12, 2013; accepted September 14, 2013. Date of publication November 1, 2013; date of current version March 6, 2014. The Associate Editor coordinating the review process was Dr. Niclas Bjorsell.

L. J. Gunn, P. G. Catlow, A. Allison, and D. Abbott are with the School of Electrical and Electronic Engineering, The University of Adelaide, Adelaide 5005, Australia (e-mail: lachlan.gunn@ adelaide.edu.au).

W. A. Al-Ashwal is with the Adelaide Radar Research Centre and School of Electrical and Electronic Engineering, The University of Adelaide, Adelaide 5005, Australia.

J. G. Hartnett is with the Institute for Photonics and Advanced Sensing, School of Chemistry and Physics, The University of Adelaide, Adelaide 5005, Australia.

Color versions of one or more of the figures in this paper are available online at http://ieeexplore.ieee.org.

Digital Object Identifier 10.1109/TIM.2013.2285796 variances $\sigma_{1}^{2}, \sigma_{2}^{2}$, and $\sigma_{3}^{2}$ are related to the measured variances $\sigma_{1,3}^{2}, \sigma_{2,3}^{2}$, and $\sigma_{1,2}^{2}$ of the time differences by

$$
\begin{aligned}
& \sigma_{1,3}^{2}=\sigma_{1}^{2}+\sigma_{3}^{2} \\
& \sigma_{2,3}^{2}=\sigma_{2}^{2}+\sigma_{3}^{2} \\
& \sigma_{1,2}^{2}=\sigma_{1}^{2}+\sigma_{2}^{2} .
\end{aligned}
$$

This system can be solved to find the original source variances

$$
\begin{aligned}
\sigma_{1}^{2} & =\frac{1}{2}\left(\sigma_{1,3}^{2}+\sigma_{1,2}^{2}-\sigma_{2,3}^{2}\right) \\
\sigma_{2}^{2} & =\frac{1}{2}\left(\sigma_{2,3}^{2}+\sigma_{1,2}^{2}-\sigma_{1,3}^{2}\right) \\
\sigma_{3}^{2} & =\frac{1}{2}\left(\sigma_{1,3}^{2}+\sigma_{2,3}^{2}-\sigma_{1,2}^{2}\right)
\end{aligned}
$$

under the assumption that the three variables are independent.

Other methods such as cross-correlation analysis [11] can fulfill the same function with improved performance, but still require at least three sources. However, any frequency measurement system will require a timebase or reference oscillator. We therefore propose that this reference also be used as one of the independent sources, allowing the application of the three-cornered hat to measurements from some dualchannel devices.

The system by Riley [12] uses a free-running reference oscillator, and provides an excellent example of the type of system that could benefit from our proposed technique. However, those by Stein et al. [13], Greenhall et al. [14], and Sandenbergh et al. [15] derive their references from a device under test (DUT), and so the additional measurement is not independent. However, as we show in Section II-A, the same techniques also apply to measurements employing certain time-interval counters and are therefore relevant to a great many laboratories.

Other measurement architectures, such as those by Hartnett [16] and by Uchino and Mochizuki [17]-[19], perform a digital phase measurement and have a similar potential for enhancement by our described method.

We examine the fundamental bases of several measurement techniques and show that, subject to certain implementation constraints, this is possible without the modification of hardware. Software modifications can be confined to a small preprocessing step before analysis.

\section{Measurement Systems}

Before discussing individual measurement systems, we first describe some quantities of interest as specified by 
IEEE Std. 1139 [20]. A sinusoidal oscillator of nominal frequency $f_{n}$ will produce a voltage

$$
V(t)=\left(V_{0}+\epsilon(t)\right) \sin \left(2 \pi f_{n} t+\phi(t)\right)
$$

where $\phi(t)$ is a phase-modulation term that incorporates both frequency offset and random fluctuations. Any deviation from the nominal frequency will manifest itself as a linearly increasing phase $\phi(t)$. It is usual then to normalize $\phi(t)$ to have units of time, giving

$$
x(t)=\frac{\phi(t)}{2 \pi f_{n}} .
$$

Its derivative is the instantaneous fractional frequency offset

$$
y(t)=\dot{x}(t)=\frac{f(t)-f_{n}}{f_{n}}
$$

where $f(t)=\dot{\phi}(t) /(2 \pi)$ is the instantaneous frequency of the oscillator. It is with these two quantities and their statistics that we are concerned, and so we now consider their measurement.

\section{A. Time-Interval Counting}

Perhaps the simplest way to measure the relative stability of a pair of oscillators is to directly measure the time between their phase edges. Consider an observer who wishes to measure the stability of a pair of oscillators with one pulse-persecond (PPS) outputs. A possible approach is to connect them to a time-interval counter, allowing measurement of their phase differences.

Let $\Delta[i]$ represent a series of these measurements, i.e., the time interval between corresponding rising phase edges of the two oscillators. We can write these intervals as the timenormalized phase difference between the two clocks [20], as

$$
\Delta[i]=x_{2}[i]-x_{1}[i] .
$$

As a result, the fractional frequency offset can be computed [20] as the derivative of $\Delta[i]$ with respect to time.

An alternative to direct time-interval measurement with a gated counter is time-tagging; time intervals can be determined by the subtraction of time-tags, thereby greatly easing multichannel measurements.

Implicit, however, is the presence of a reference oscillator that is relatively stable over the time intervals in question. This third oscillator provides the possibility to make three comparisons. The observer may therefore divide the reference oscillator frequency to $1 \mathrm{~Hz}$ - or more generally to the nominal frequency $f_{n}$ of the DUTs - and tag its edges $u[i]$ as in Fig. 1 . This allows the measurement of three relative phases as needed by the three-cornered hat: one between the DUTs, and one between each DUT and the timebase.

However, because the phase edges of the divided signal are coincident with each $\left(f_{r} / f_{n}\right)$ th phase edge of the timebase, the time-tags $u[i]$ will not display noise from the reference. Instead, the rising edges will occur on each $\left(f_{r} / f_{n}\right)$ th count of the time-tagger, subject to noise from the divider. In units of normalized phase, we may then write the time-tags as

$$
u[i]=\frac{i}{f_{n}}+\delta D[i]
$$

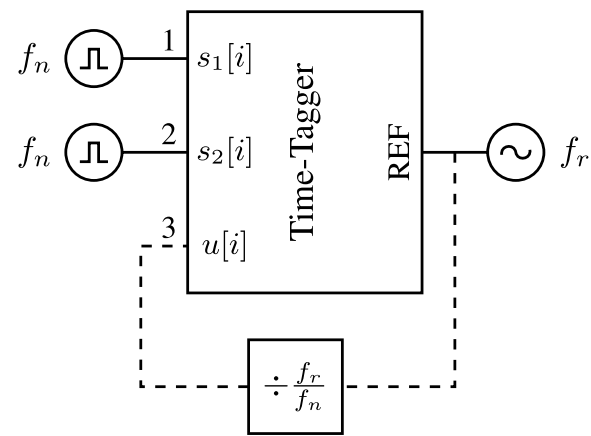

Fig. 1. Hypothetical time-tagging system for frequency stability measurement. By dividing the reference oscillator to the same nominal frequency as the devices under test, one produces a third signal for comparison. As the phase edges of the new signal are coincident with every $\left(f_{r} / f_{n}\right)$ th phase edge of the reference, the time-tags are known, except for the noise of the divider.

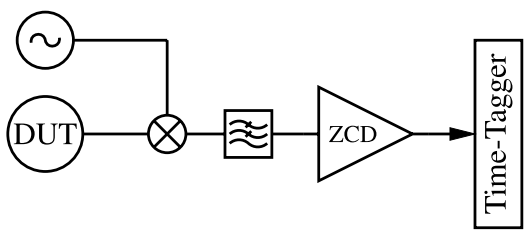

Fig. 2. Heterodyne method mixing the DUT with a reference oscillator and producing a frequency at the output that is equal to the difference in the frequencies of the two inputs. A zero-crossing detector is used to find the rising phase edge of the signal, which is then time-tagged.

where $\delta D[i]$ is the time error introduced by the divider. In the ideal case where the divider is noiseless

$$
u[i]=\frac{i}{f_{n}} .
$$

This implies that one need not physically divide and time-tag the reference oscillator, but can instead simply insert uniformly spaced time-tags into the data, producing results identical to those obtained with a noiseless divider. Note that, while the time-tags $u[i]$ are known exactly, fluctuations of the reference oscillator will cause variation in the times to which they refer. The same applies to the measured time-tags $s_{1}[i]$ and $s_{2}[i]$, so we may write those time-tags in terms of the true phases

$$
\begin{aligned}
& s_{1}[i]=x_{1}[i]+\delta R[i] \\
& s_{2}[i]=x_{2}[i]+\delta R[i]
\end{aligned}
$$

where $\delta R[i]$ represents the phase error added by the reference.

We subtract the three time-tags from one another to find the relative phases

$$
\begin{aligned}
\Delta_{1,2}[i] & =x_{1}[i]-x_{2}[i] \\
\Delta_{1, R}[i] & =x_{1}[i]-u[i]+\delta R[i] \\
\Delta_{2, R}[i] & =x_{2}[i]-u[i]+\delta R[i] .
\end{aligned}
$$

As $u[i]$ is deterministic, its variance is zero. The measured variances are therefore

$$
\begin{aligned}
\sigma_{1,2}^{2} & =\sigma_{1}^{2}+\sigma_{2}^{2} \\
\sigma_{1, R}^{2} & =\sigma_{1}^{2}+\sigma_{R}^{2} \\
\sigma_{2, R}^{2} & =\sigma_{2}^{2}+\sigma_{R}^{2}
\end{aligned}
$$


allowing application of the three-cornered hat with standard methods such as in [10] or [11].

This technique is not restricted to systems that operate by time-tagging signal edges, but wherever it is possible to determine the relative phase between DUTs and a common reference oscillator. We now demonstrate that this is the case for heterodyne measurements, and in particular the dual-mixer time-difference (DMDT) method.

\section{B. Heterodyne Method}

The heterodyne method [21] mixes two sinusoids to provide a measurement of their frequency difference. The mixture is low-pass-filtered to extract the difference frequency, which can be measured with a counter.

Let us denote the reference and device signals as

$$
\begin{aligned}
& v_{r}(t)=\cos \left(2 \pi f_{n} t+\phi_{r}(t)\right) \\
& v_{d}(t)=\cos \left(2 \pi f_{n} t+\phi_{d}(t)\right)
\end{aligned}
$$

where $f_{n}$ is the nominal frequency of the two oscillators, and $\phi_{r}(t)$ and $\phi_{d}(t)$ are phase-modulation terms that incorporate both frequency offset and random fluctuations.

The output of the low-pass filter is given by

$$
v_{o}(t)=\frac{1}{2} \cos \left(\phi_{d}(t)-\phi_{r}(t)\right) .
$$

A frequency offset will manifest itself as a linearly increasing phase difference, resulting in a sinusoidal $v_{o}(t)$. However, one may also choose to interpret the zero-crossings as those points where the phase difference is an integer multiple of $2 \pi$. If the reference frequency is chosen to be slightly less than the nominal frequency $f_{n}$ of the device (suppose by $f_{b}$ ), then each zero-crossing may be interpreted as a point at which the phase difference has increased by an entire cycle. One may then use interpolation to estimate the fractional number of elapsed cycles $N(t)$ at uniformly spaced points and so determine the time-scaled phase difference between the two oscillators

$$
x_{d}[i]=\frac{N\left(t_{i}\right)-t_{i} f_{b}}{f_{n}} .
$$

The second term is taken from [14] and accounts for the frequency offset $f_{b}$ of the reference from the nominal frequency. Its value $t_{i} f_{b}$ is the fractional number of cycles $N_{b}\left(t_{i}\right)$ that would have passed if a perfect oscillator-from the perspective of the reference-were connected instead of the DUT.

We therefore see that heterodyning provides a method by which the difference in phase between two oscillators may be determined. While this is not on its own enough to characterize the DUT, except where the reference is of negligible or known identical instability, it is used as the basis for a more accurate technique, namely the DMTD method.

\section{DMDT Method}

A popular technique for high-resolution measurement of time stability is the DMTD method [21], [22], shown in Fig. 3.

The fundamental principle of the DMTD method is that downconversion of two signals with the same local oscillator

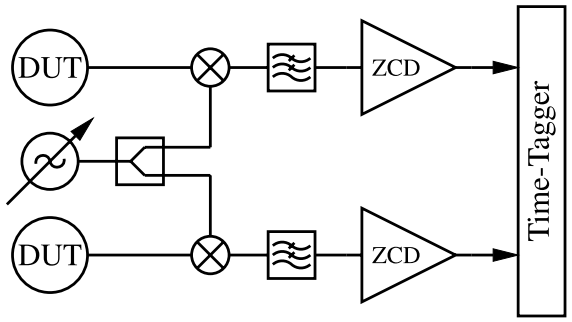

Fig. 3. The dual-mixer time difference method allows the measurement of phase error with tremendous resolution. The oscillators under test are coherently downconverted, reducing their frequency but maintaining their relative phase. This increases the time difference between their rising edges and so allows greater accuracy. Zero-crossing detectors are used to locate the rising edges of each signal, which are then time-tagged. Alternatively, methods such as that by Uchino and Mochizuki [17]-[19] digitize the downconverted signals and perform the phase comparison digitally.

preserves their relative phase. By reducing their frequencies from several megahertz to a few hertz or kilohertz, the time between zero-crossings is amplified by the same factor by which frequency is reduced.

As with the simple event measurements described earlier, many instruments such as in [12], [13], and [22] use a timeinterval counter to measure the difference in the times of arrival of the corresponding edges. This can then be used to compute the difference in phase between the two DUTs. While this allows the use of an off-the-shelf time-interval counter, it discards all information on the relative phase of the devices and the reference oscillator.

Greenhall et al. [14] interpret this method, instead, as two heterodyne measurements against a single oscillator. This provides two simultaneous phase measurements, allowing the system to be fully characterized as in Section II-A. That is to say, if $x_{1}[i], x_{2}[i]$, and $x_{r}[i]$ represent the phases of the two DUTs and the reference, respectively, we measure

$$
\begin{aligned}
& \Delta_{1, r}[i]=x_{1}[i]-x_{r}[i] \\
& \Delta_{2, r}[i]=x_{2}[i]-x_{r}[i]
\end{aligned}
$$

and then compute

$$
\begin{aligned}
\Delta_{1,2}[i] & =x_{1}[i]-x_{2}[i] \\
& =\Delta_{1, r}[i]-\Delta_{2, r}[i] .
\end{aligned}
$$

With this realization, it becomes clear that the use of timetagging allows the DMTD method to measure all three phase offsets in the system. If the common oscillator is independent of the DUTs, this means that the individual stabilities can be determined by methods previously discussed.

As there already exist time-tagging DMTD systems, such as that of Greenhall et al. [14], we now turn to the question of whether these additional measurements can be performed by inserting time-tags as a third channel, as demonstrated in Section II-A. These systems can compute all differences $\Delta_{p, q}[i]$ as in (21). Substituting (18) into (21), we see that this measurement can be written as

$$
\Delta_{p, q}[i]=\frac{N_{p}\left(t_{i}\right)-N_{q}\left(t_{i}\right)}{f_{n}} .
$$


If one introduces time-tags $u_{r}[i]$ so that the number of elapsed cycles interpolates to

$$
N_{r}\left(t_{i}\right)=t_{i} f_{b}
$$

then the system will automatically recover all of the $\Delta_{p, r}$ introduced previously. An oscillator at the nominal frequency perfectly aligned with the reference will produce a beat signal of frequency $f_{b}$, and so

$$
u_{r}[i]=\frac{i}{f_{b}} .
$$

As this is linear in $i$, the number of cycles $i$ elapsed at time $t_{i}$ can be found by allowing $i$ to be real and solving (25) to find

$$
i=f_{b} t_{i}
$$

which is of the form that we desire. Therefore, by inserting time-tags according to (25) one adds an additional source to those existing multichannel DMTD systems with a freerunning reference oscillator.

\section{Simulation}

We now demonstrate the utility of these methods by applying the three- and four-cornered hat to simulated data, showing that the extra oscillator can provide a meaningful enhancement to the measurement system. While the four-cornered hat is by no means new, we wish to determine the conditions under which our technique will provide an enhancement. We first consider the measurement of white noise. We simulate four uncorrelated noise processes $X_{1}[i], X_{2}[i], X_{3}[i]$, and $X_{R}[i]$. The last one is varied, while the first three have fixed variances of $4 \cdot 10^{-18}, 9 \cdot 10^{-18}$, and $10^{-18}$, respectively. We then compute the differences $\Delta_{p, q}[i]$ and their normal variances $\sigma_{p, q}^{2}$, and so use the three-cornered hat [10] to reconstruct the individual device variances $\sigma_{i}^{2}$. We then compare the results with the true noise variances and thus determine the error of the estimation process.

If the time differences between the reference and device oscillators are used as well, we have more potential measurements than we have sources and can now solve the M-cornered-hat problem [23]. We therefore use least squares to solve the resulting system of linear equations. This admits the possibility of weighting the various measurements. We choose the weights as in [23] to be inversely proportional to the magnitude of the measured variance, as the estimated variances are expected to have a $\chi^{2}$ distribution and so a variance proportional to the true value.

The results are shown in Fig. 4(a) and (b). As subtraction of the time-tags removes the effect of the reference oscillator, the error does not vary with its noise when the traditional method is used.

However, our proposed method causes the uncertainty to vary with the stability of the reference. When the reference is stable, these extra measurements allow a significantly lower uncertainty than with only three comparisons. As the reference becomes noisier, the estimate is degraded, though, as this occurs, the variances of its measurements increase and so are weighted less and less, eventually becoming negligible.

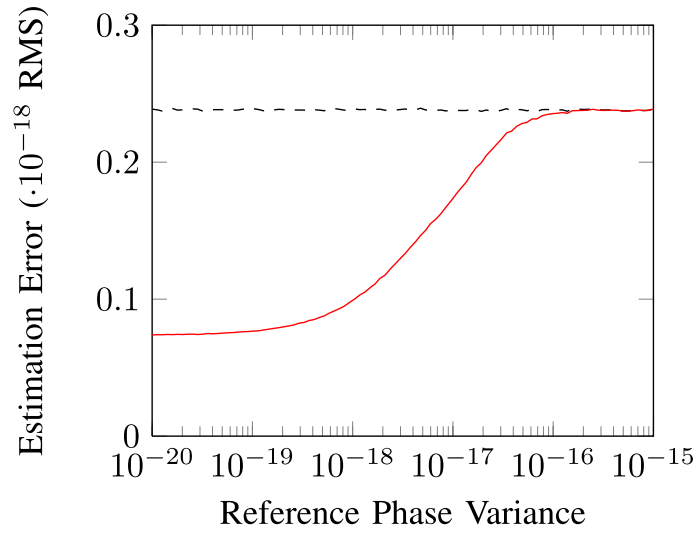

(a)

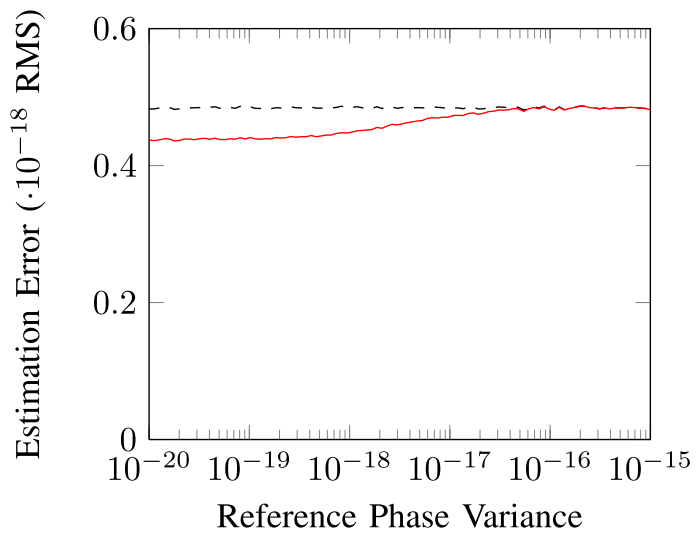

(b)

Fig. 4. Effect of reference noise on the estimation of noise variances. The variances being measured are $4 \cdot 10^{-18}, 9 \cdot 10^{-18}$, and $10^{-18}$. If the reference is not considered (dashed), then its variance will not affect the uncertainty of the estimate. If the variances of the time-tags are used in the calculation (solid), the uncertainty reduces. The relative improvement is greater when small variances are being measured as in Fig. 4. In either case, the enhancement starts to appear when the noise of the reference falls below ten times that of the noisiest device under test. (a) Small measured noise variance. (b) Large measured noise variance.

TABLE I

Noise Parameters for Simulated Oscillators Shown IN Fig. 6 18000 Simulated Samples. The Processes With CoefFicients

Below Are Added Together to Form the Normalized PHASE FOR THE RESPECTIVE OSCILLATORS

\begin{tabular}{|c|c|c|}
\hline & Stable Source & Other Sources \\
\hline White PM & $2 \cdot 10^{-9}$ & $5 \cdot 10^{-9}$ \\
White FM & $1 \cdot 10^{-12}$ & $2 \cdot 10^{-10}$ \\
RW FM & $1 \cdot 10^{-11}$ & $12 \cdot 10^{-11}$ \\
\hline
\end{tabular}

The advantage is greatest when measuring small variances, as in Fig. 4(a). The additional measurements tend to provide a level of averaging, reducing the $\chi^{2}$ error in the variances of the oscillators under test. When the individual variance of interest is large in comparison with the other oscillators, as in Fig. 4(b), the reduction in error is far less.

We next consider the more practical case of measurements of the Allan variance [24], [25], which is equal to half the variance of the difference between consecutive 


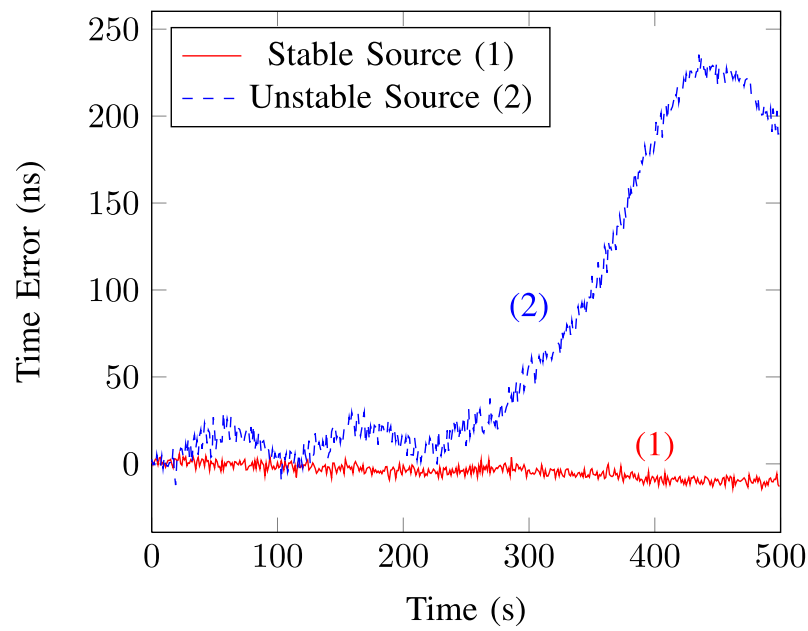

(a)

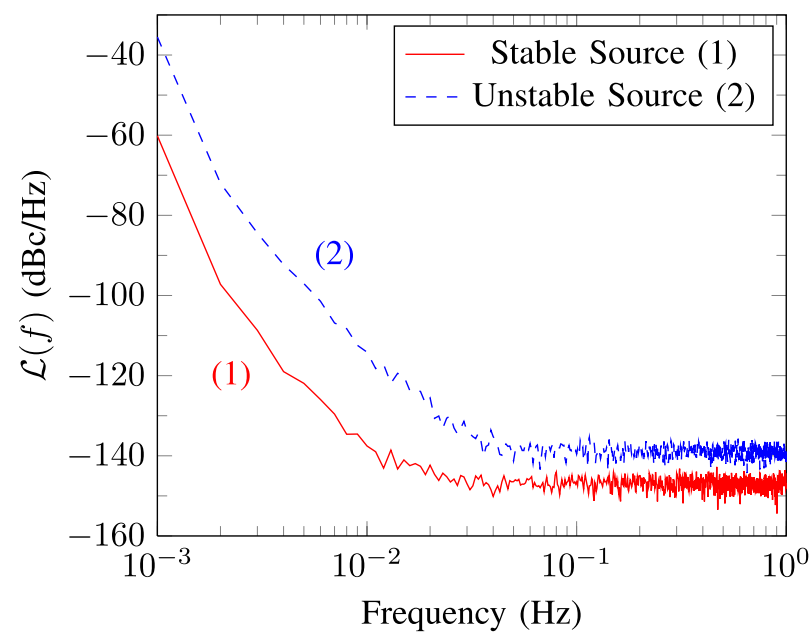

(b)

Fig. 5. Processes described in Table I. The unstable sources have far more frequency drift, causing the rapid deviation visible in Fig. 5. The phase noise of the two oscillators is shown in Fig. 5. Beyond $100 \mathrm{mHz}$, the processes are dominated by white noise. (a) Time error. (b) SSB phase Noise.

average frequency measurements. Using the overlapping form as given by Riley [21], we simulate four random processes, each the sum of white phase noise (white PM), white frequency noise (white FM), and random-walk frequency noise (RW FM) processes, using the method of Kasdin and Walter [26]. The scaling of each is shown in Table I; these dimensionless values are chosen so as to produce Allan variances with general characteristics similar to those shown in [21] and relative magnitudes near to the limits of the threecornered hat's utility. One of the sources is considerably more stable than the others, as is the case when characterizing the first prototype of a new and more stable frequency standard. A small segment of a simulation of the processes is shown in Fig. 5, along with the measured phase noise.

The Allan variance of each process is calculated, and the three-cornered hat is used to compute the Allan variance of the stable source, as shown in Fig. 6. Then, the fourth source is added to the system in order to solve the four-cornered-hat

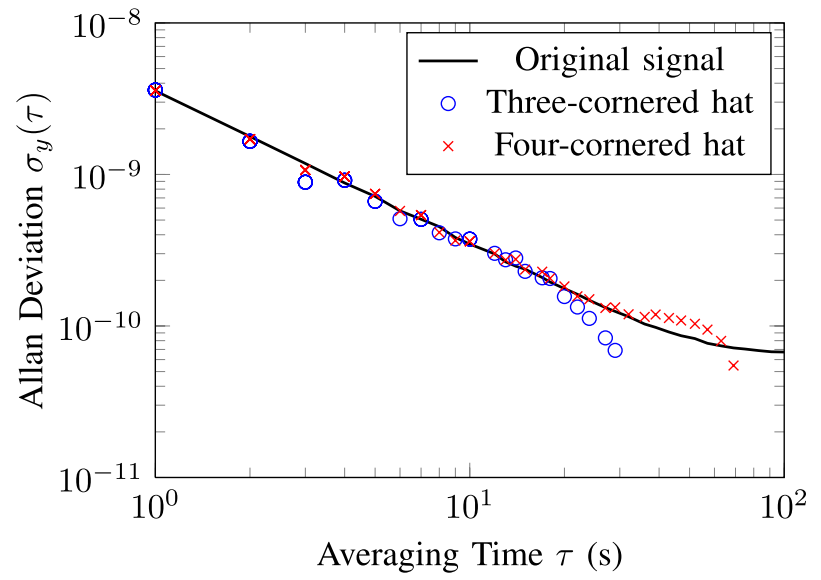

Fig. 6. Allan deviation $\sigma_{y}(\tau)$ of the more stable source computed with and without an extra oscillator, as would be introduced by the method described. The additional measurements result in less uncertainty, particularly at longer averaging times.

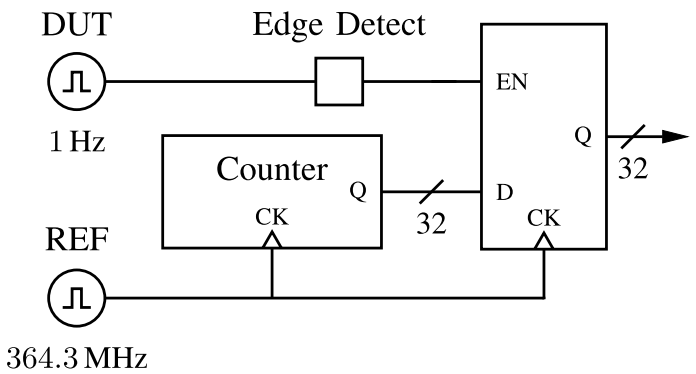

Fig. 7. Single time-tagging channel from our experimental setup. The full design has two such channels, with the reference and counter shared between them. The reference is phase-locked to an uncompensated crystal oscillator, and the register output transferred to a PC via USB upon each edge. Ambiguity caused by overflow of the counter is resolved in software.

problem-also shown in Fig. 6. We see that the additional source provides more stable estimates of the Allan variance even at long averaging times, and extends the usable range of averaging times by a substantial degree. This is significant because it demonstrates that the introduction of an additional source by the proposed method will substantially improve the ability of existing systems to resolve the behavior of highly stable oscillators over short periods.

\section{EXPERIMENT}

We verify this technique experimentally by measuring the frequency stabilities of a pair of temperature-compensated crystal oscillators (TCXOs) with a field-programmable gate array (FPGA), as shown in Fig. 7. The FPGA development board includes a crystal oscillator that is used as a timebase. This mirrors the configuration of a commercial dual-channel frequency counter such as [27], though the noisy reference oscillator reduces its accuracy substantially. The TCXOs being measured include a divider to produce a $1-\mathrm{Hz}$ output.

The FPGA time-tags the DUT edges, which are transferred to a PC via USB. We again compute the three differences $\Delta_{p, q}[i]$, allowing the calculation of the combined Allan variances. We then use the three-cornered hat to find the individual Allan deviations, as shown in Fig. 8. 


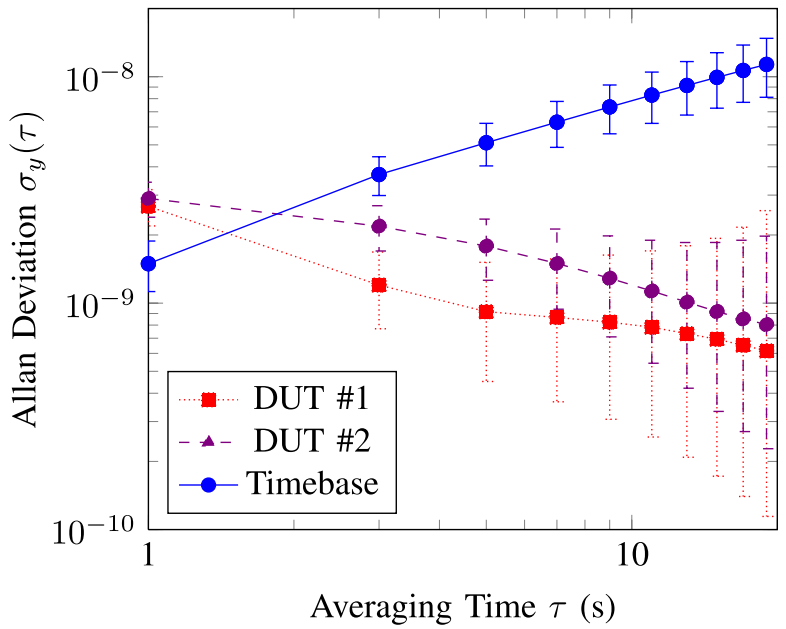

Fig. 8. Allan deviations $\sigma_{y}(\tau)$ of a pair of TCXOs measured by an FPGA and standard crystal oscillator. The error bars are $95 \% \chi^{2}$ confidence intervals [28], with the effective degrees of freedom determined using [21] and [9]. Measured over $6.5 \mathrm{~h}$, the three phase errors $\Delta_{p, q}[i]$ are constructed and the Allan deviations computed. The stability of the individual TCXOs can be seen, though their uncertainties are quite large because of the relatively noisy timebase. The two TCXOs have similar characteristics as one would expect.

The results for the two TCXOs are quite similar, as expected. At longer averaging times, the results begin to degrade because of the relatively noisy timebase and small sample size. Despite this, the results show that we are able to separate the fractional frequency stabilities of the individual devices using only a dual-channel time-tagging instrument. This feat demonstrates that current instruments can provide such capability with the injection of additional timestamps.

\section{DISCUSSION}

As many DMTD systems derive their reference signal from an input channel, the assumption of independence required by the three-cornered-hat technique will often be violated. However, wherever a free-running reference oscillator is used, the technique discussed here allows individual device variances to be estimated with a two-channel DMTD system.

Perhaps the greatest benefit occurs when using a timeinterval counter as in Section II-A. Those time interval counters that can produce a series of timestamps-the Agilent 53230A frequency counter [27], for example — can potentially be repurposed to measure the stability of their DUTs even when they approach or slightly exceed that of the internal timebase. Such devices are normally dual-channel and so incapable of performing three-cornered-hat measurements without this technique.

As the proposed method takes the form of a preprocessing step that occurs prior to any other analysis, it can also be applied to existing data from time-tagging instruments. This provides the opportunity for the reanalysis of previous experiments with reduced uncertainty.

\section{CONCLUSION}

We have demonstrated that two common forms of frequency stability measurement, namely time interval counting and the DMTD method, when implemented with a time-tagging system, capture not only the relative stabilities of the DUTs but also that of the reference oscillator, allowing the use of the three-cornered hat in cases which would otherwise require an additional oscillator and measurement channel. We have shown that this instability can be accessed by inserting uniformly spaced time-tags into one's data. We have demonstrated this experimentally by measuring the Allan variance of a pair of oscillators using a dual-channel time-tagging system.

\section{ACKNOWLEDGMENT}

The authors would like to thank Prof. D. A. Gray for his assistance during an earlier phase of the project.

\section{REFERENCES}

[1] B. Parkinson and S. Gilbert, "NAVSTAR: Global positioning systemTen years later," Proc. IEEE, vol. 71, no. 10, pp. 1177-1186, Oct. 1983.

[2] W. Lindsey, F. Ghazvinian, W. Hagmann, and K. Dessouky, "Network synchronization," Proc. IEEE, vol. 73, no. 10, pp. 1445-1467, Oct. 1985.

[3] T. D. Carr, M. A. Lynch, M. P. Paul, G. W. Brown, J. May, N. F. Six, et al., "Very long baseline interferometry of Jupiter at $18 \mathrm{MHz}$," Radio Sci., vol. 5, no. 10, pp. 1223-1226, Oct. 1970.

[4] N. Nand, J. Hartnett, E. Ivanov, and G. Santarelli, "Ultra-stable very-low phase-noise signal source for very long baseline interferometry using a cryocooled sapphire oscillator," IEEE Trans. Microw. Theory Tech., vol. 59, no. 11, pp. 2978-2986, Nov. 2011.

[5] D. Dravins, S. LeBohec, H. Jensen, and P. D. Nuñez, "Optical intensity interferometry with the Cherenkov telescope array," Astroparticle Phys., vol. 43, pp. 331-347, Mar. 2013.

[6] J. G. Hartnett and A. N. Luiten, "Colloquium: Comparison of astrophysical and terrestrial frequency standards," Rev. Modern Phys., vol. 83, pp. 1-9, Jan. 2011.

[7] M. Weiss, "Synchronisation of bistatic radar systems," in Proc. IEEE IGARSS, vol. 3. Sep. 2004, pp. 1750-1753.

[8] G. Krieger and A. Moreira, "Spaceborne bi- and multistatic SAR: Potential and challenges," IEE Proc. Radar, Sonar Navigat., vol. 153, no. 3, pp. 184-198, 2006.

[9] C. Ekstrom and P. Koppang, "Error bars for three-cornered hats," IEEE Trans. Ultrason., Ferroelectr. Freq. Control, vol. 53, no. 5, pp. 876-879, May 2006.

[10] J. Gray and D. Allan, "A method for estimating the frequency stability of an individual oscillator," in Proc. 28th Annu. Symp. Freq. Control, 1974, pp. 243-246.

[11] A. Lepek and F. Walls, "Cross correlation analysis improves time domain measurements," in Proc. 47th IEEE Int. Freq. Control Symp., Jun. 1993, pp. $312-320$.

[12] W. J. Riley. (2013, Apr. 4). A Small Dual Mixer Time Difference (DMTD) Clock Measuring System [Online]. Available: http://www.wriley.com/A Small DMTD System.pdf

[13] S. Stein, D. Glaze, J. Levine, J. Gray, D. Hilliard, D. Howe, et al., "Performance of an automated high accuracy phase measurement system," in Proc. 36th Annu. Symp. Freq. Control, Jun. 1982, pp. 314-320.

[14] C. A. Greenhall, A. Kirk, and R. L. Tjoelker, "Frequency standards stability analyzer for the deep space network," JPL, Caltech, CA, USA, Tech. Rep. 42-169, 2007.

[15] J. S. Sandenbergh, M. Inggs, and W. A. Al-Ashwal, "Evaluation of coherent netted radar carrier stability while synchronised with GPS-disciplined oscillators," in Proc. IEEE Radar Conf., May 2011, pp. 1100-1105.

[16] J. G. Hartnett, S. R. Parker, and E. N. Ivanov, "Novel frequency transposing measurement technique for oscillator comparisons," Electron. Lett., vol. 49, pp. 947-949, Jul. 2013.

[17] M. Uchino and K. Mochizuki, "Frequency stability measuring technique using digital signal processing," Electron. Commun. Jpn. Part I, Commun., vol. 87, no. 1, pp. 21-33, Jan. 2004.

[18] K. Mochizuki, M. Uchino, and T. Morikawa, "Frequency-stability measurement system using high-speed ADCs and digital signal processing," IEEE Trans. Instrum. Meas., vol. 56, no. 5, pp. 1887-1893, Oct. 2007.

[19] S. Yanagimachi, K. Hagimoto, and T. Ikegami, "Analysis of truncation error for dual-mixer time-difference measurement system using discrete Fourier transformation," Jpn. J. Appl. Phys., vol. 52, no. 3, pp. 036601-1-036601-5, Feb. 2013. 
[20] IEEE Standard Definitions of Physical Quantities for Fundamental Frequency and Time Metrology—Random Instabilities, IEEE Standard 1139-1999, 1999.

[21] W. J. Riley, "Handbook of frequency stability analysis," Ph.D. dissertation, U.S. Dept. Commerce, Nat. Inst. Stand. Technol., Boulder, CO, USA, 2008

[22] D. Allan and H. Daams, "Picosecond time difference measurement system," in Proc. 29th Annu. Symp. Freq. Control, 1975, pp. 404-411.

[23] C. A. Greenhall, "Likelihood and least-squares approaches to the M-cornered hat," in Proc. 19th Annu. PTTI, Dec. 1987, pp. 1-9.

[24] D. W. Allan, "Statistics of atomic frequency standards," Proc. IEEE, vol. 54, no. 2, pp. 221-230, Feb. 1966

[25] S. Stein, "The Allan variance-Challenges and opportunities," IEEE Trans. Ultrason., Ferroelectr. Freq. Control, vol. 57, no. 3, pp. 540-547, Mar. 2010.

[26] N. Kasdin and T. Walter, "Discrete simulation of power law noise," in Proc. IEEE 46th Аnnu. Symp. Freq. Control, May 1992, pp. 274-283.

[27] Agilent 53220A/230A Frequency Universal Counter/Timer Programmer's Reference, NASA, Washington, DC, USA, 2010.

[28] E. Kreyszig, Advanced Engineering Mathematics, 9th ed. New York, NY, USA: Wiley, 2006.

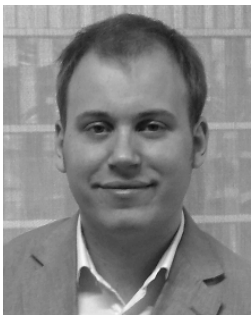

Lachlan J. Gunn (S'08) received the B.Eng. (Hons.), B.M., and C.Sc. (Pure) degrees from the University of Adelaide, Adelaide, Australia, in 2012, and is currently pursuing the Ph.D. degree under the supervision of D. Abbott and A. Allison.

His current research interests include informationtheoretic security and the use of stochastic signal processing for characterization of nonlinear systems.

Mr. Gunn received the 2012 J. Mazumdar Prize in Engineering and Mathematics and the four DSTO Scholarships in Radar Technology from 2009 to 2012. In 2013, he was granted an Australian Postgraduate Award.

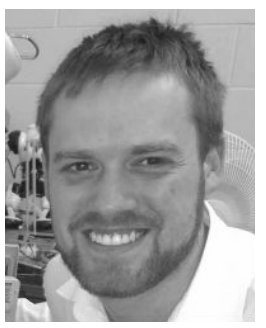

Peter G. Catlow received the B.Eng. (Hons.) degree from the University of Adelaide, Adelaide, Australia in 2012.

$\mathrm{He}$ is involved in scientific outreach, promoting electronic engineering among the general public. His current research interests include control theory, radar for navigation and detection, and communications.

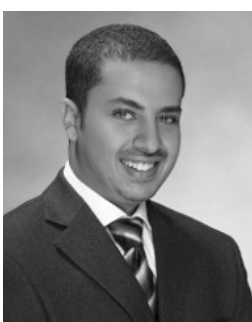

Waddah A. Al-Ashwal (S'11-M'12) received the B.S. (Hons.) degree in electrical engineering from Sana'a University, Sana'a, Yemen, the M.Sc. (Hons.) degree in digital communications and microwaves from the University of Manchester, Manchester, $\mathrm{U} . \mathrm{K}$, and the Ph.D. degree in electronic engineering from University College London, London, U.K., under the supervision of $\mathrm{K}$. Woodbridge and $\mathrm{H}$. Griffiths, in 2003, 2007, and 2011, respectively.

$\mathrm{He}$ was a Wireline Field Engineer with Schlumberger S.A., from 2003 to 2005 . From 2005 to 2006, he was a Teaching Assistant with Taiz University, Ta'izz, Yemen. Since 2011, he has been a Research Fellow with the Adelaide Radar Research Centre, School of Electrical and Electronic Engineering, University of Adelaide, Adelaide, Australia. His current research interests include radar system design and signal processing, particularly in relation to polarimetric radar, radar phenomenology, and multistatic radar system.

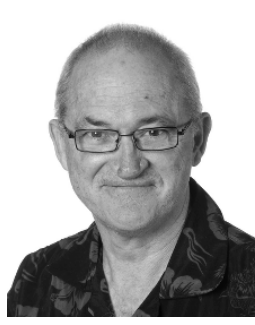

John G. Hartnett received the B.Sc. and Ph.D. (Hons.) degrees in physics from the University of Western Australia (UWA), Crawley, Australia.

He served as a Research Professor with the Frequency Standards and Metrology Research Group, UWA. In 2013, he was an Australian Research Council Discovery Outstanding Researcher Award Fellowship based within the Institute for Photonics and Advanced Sensing, and the School of Chemistry and Physics, University of Adelaide, Adelaide, Australia, where he is currently an Associate Professor. His current research interests include planar metamaterials, development of ultrastable microwave oscillators based on sapphire resonators, and tests of fundamental theories of physics such as special and general relativity using precision oscillators.

Prof. Hartnett was a recipient of the 2010 IEEE UFFC Society W. G. Cady Award. He was a co-recipient of the 1999 Best Paper Award presented by the Institute of Physics Measurement Science and Technology.

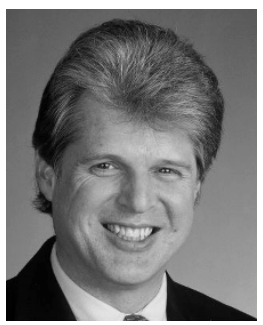

Andrew Allison received the B.Sc. degree in mathematical sciences, the B.Eng. (Hons.) degree in computer systems engineering, and the Ph.D. degree in electrical and electronic engineering from the University of Adelaide, Adelaide, Australia, in 1978, 1995, and 2009, respectively, under the supervision of D. Abbott and C. E. M. Pearce.

He was with Barrett Brothers, Adelaide, from 1976 to 1977 , as a Laboratory Technician, involved in performing chemical assays. From 1980 to 1981, he was with the Commonwealth Scientific and Industrial Organization, Urrbrae, Australia, in the area of high pressure liquid chromatography, analysis of infrared spectroscopy data, and analysis of radioactive assays of DNA recombination. From 1981 to 1995, he held various positions, mainly in the area of local area networks, at what came to be known as the Telstra Corporation, Melbourne, Australia. Since 1995, he has been a Lecturer with the School of Electrical and Electronic Engineering, University of Adelaide. His current research interests include probability, statistics and estimation, control theory, communication theory, and diffusion processes.

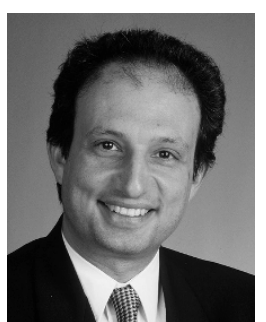

Derek Abbott (M'85-SM'99-F'05) was born in London, U.K., in 1960. He received the B.Sc. (Hons.) in physics from Loughborough University, Loughborough, U.K., in 1982, and the Ph.D. degree in electrical and electronic engineering from the University of Adelaide, Adelaide, Australia, in 1995, under the supervision of K. Eshraghian and B. R. Davis.

He was a Research Engineer with the GEC Hirst Research Centre, London, from 1978 to 1986. From 1986 to 1987, he was a VLSI Design Engineer with Austek Microsystems, Adelaide. Since 1987, he has been with the University of Adelaide, where he is currently a Full Professor with the School of Electrical and Electronic Engineering. He holds over 800 publications/patents and has been an invited speaker at over 100 institutions. He has co-edited Quantum Aspects of Life (Imperial College Press), and has co-authored Stochastic Resonance (Cambridge University Press) and Terahertz Imaging for Biomedical Applications (Springer-Verlag). His research programs span a number of areas of stochastics, game theory, photonics, biomedical engineering, and computational neuroscience. His current research interests include multidisciplinary physics and electronic engineering applied to complex systems.

Prof. Abbott is a fellow of the Institute of Physics (IOP). He has received a number of awards including the South Australian Tall Poppy Award for Science in 2004, the Premier's SA Great Award in Science and Technology for outstanding contributions to South Australia in 2004, and the Australian Research Council Future Fellowship in 2012. He has served as an Editor and Guest Editor for a number of journals, including the IEEE JOURNAL OF SOLID-STATE CIRCUITS, Journal of Optics B (IOP), Microelectronics Journal (Elsevier), and is currently on the editorial boards of the PROCEEDINGS OF THE IEEE, the IEEE PHOTONICS JOURNAL, and PLOS ONE. 